\title{
Perturbation hydrique et altération des surfaces dans les aiguilles d'épicéas (Picea abies L) du Jura en fonction de leur âge et de l'état de dépérissement des arbres
}

\author{
PM Badot 1, JP Garrec 2 \\ 1 Faculté des sciences et des techniques, Place Leclerc, \\ laboratoire de botanique, 25030 Besançon Cedex; \\ 2 INRA, centre de recherches forestières, laboratoire d'étude de la pollution atmosphérique, \\ Champenoux, 54280 Seichamps, France
}

(Reçu le 14 mai 1990; accepté le 14 août 1990)

\begin{abstract}
Résumé - L'étude du contenu hydrique des aiguilles et l'observation en microscopie électronique à balayage des surfaces foliaires ont été effectuées sur un large échantillon d'épicéas sains et d'épicéas dépérissants afin de caractériser l'actuel dépérissement de cette essence dans le Jura français. Les variations de la teneur moyenne en eau sont étroitement liées à l'état de défoliation des arbres : les aiguilles des arbres fortement défoliés présentent une réduction sensible de la teneur moyenne en eau par rapport aux aiguilles des arbres non défoliés. Les aiguilles des arbres dépérissants sont également caractérisées par des modifications du nombre et de la structure des cires épicuticulaires et des cires substomatiques. A un âge donné, les aiguilles des arbres dépérissants présentent des teneurs en eau voisines de celles enregistrées un an plus tard chez les arbres sains. Le dépérissement des épicéas du Jura français se traduirait ainsi par une sénescence prématurée des aiguilles.
\end{abstract}

Picea abies / dépérissement forestier / teneur en eau / cire cuticulaire / sénescence

Summary - Perturbation of water content and surface alteration in needles of spruce trees (Picea abies $\mathrm{L}$ ) from the French Jura according to their age and to the health status of the trees. Measurements of needle water content and scanning electron microscopy studies of leaf surfaces were carried out on a large number of declining and healthy spruce trees in order to characterize the decline of this species in the French Jura. Variations in water content were closely correlated with the health status of the trees: needles of damaged trees displayed a marked decrease in leaf water content. Moreover, the number and structure of the epicuticular waxes and also the substomatal waxes were modified. At a given age, the needles of declining trees had the same water content as those displayed one year later by needles of the healthy trees. Thus, the decline of spruce trees in the French Jura appears to correspond to an early ageing of the needles.

Picea abies / forest decline / water content / cuticular wax / ageing 


\section{INTRODUCTION}

Les épicéas des forêts jurassiennes, comme de nombreuses autres essences forestières, sont affectés depuis quelques années d'importants phénomènes de dépérissement (Ministère de l'Agriculture, 1988; Badot et Badot, 1989). Dans cette région, il n'existe pas de relation entre le dépérissement et les caractéristiques pédologiques des stations (Bruckert et Tan, 1986). Ceci est confirmé par le fait que le dépérissement de l'épicéa dans le Jura ne se traduit pas par l'apparition de troubles nutritionnels (Badot et al, 1988), à l'inverse de ce qui a été rapporté pour d'autres massifs forestiers européens (Rehfuess, 1987; Landmann et al, 1987). Récemment, il a été montré que les sécheresses qui ont affecté l'Europe au cours des dernières années sont impliquées dans le dépérissement du sapin dans les Vosges (Lévy et Becker, 1987; Becker, 1989). Une telle conclusion a pu également être avancée concernant le déclin de l'épicéa dans le Jura : de mauvaises conditions d'alimentation hydrique ont contribué à l'apparition du phénomène en Franche-Comté (Badot et al, 1990). A ce stade des recherches, il apparaît important de déterminer si les variations de teneur en eau des aiguilles sont un symptôme constant chez les arbres malades et si elles sont un indicateur de dépérissement. Parallèlement, nous avons entrepris une étude détaillée en microscopie électronique à balayage des surfaces foliaires afin d'établir une corrélation entre d'éventuelles altérations des cuticules et le dépérissement des arbres.

\section{MATÉRIEL ET MÉTHODES}

\section{Matériel végétal et prélèvements}

Les prélèvements ont été effectués dans une région située entre les communes de Levier,
Frasne et Pontarlier (Doubs, France). Les arbres étudiés sont localisés dans 7 stations situées à proximité d'un même transect et constituent un échantillon représentatif (altitude, types phytosociologiques, etc.) de l'ensemble du massif.

La notation des arbres a été effectuée par les agents spécialisés de l'ONF selon le système à 5 degrés retenu dans le programme DEFORPA (Buffet, 1987). Dans chacune des 7 stations, les prélèvements ont porté sur 8 épicéas sains de classe 1, c'est-à-dire présentant moins de $10 \%$ de perte de feuillage et sur 8 épicéas dépérissants de classe 3 , dont la défoliation est comprise entre 25 et $60 \%$. Les arbres sont âgés de 70 à 110 ans. La collecte a concerné 4 générations d'aiguilles (années 1984 à 1987). La récolte des échantillons végétaux a été effectuée au mois d'octobre 1987, c'est-à-dire en période de repos végétatif, au moment où l'activité métabolique des aiguilles est la plus stable (Guehl, 1985). Les prélèvements ont été effectués sur des rameaux situés à $4 \mathrm{~m}$ de la cime au moyen d'échelles installées sur les arbres. Cette manière d'opérer précise mais lourde à mettre en œuvre a été préférée à la traditionnelle méthode de prélèvement par tir au fusil, car des résultats antérieurs (Badot et al, 1988) ont montré que certaines caractéristiques physiologiques des aiguilles et notamment leur teneur en eau étaient fonction de leur position dans les houppiers.

\section{Contenu hydrique}

Les aiguilles sont sectionnées au niveau de leur point d'insertion et sont immédiatement placées, par lot de 15, dans des tubes étanches préalablement tarés. La mesure de la masse fraîche (MF) est réalisée dès le retour au laboratoire. La détermination de la masse sèche (MS) est effectuée après 2 semaines de séjour dans une étuve à $90^{\circ} \mathrm{C}$. Dans ces conditions, nous avons vérifié que la masse des aiguilles devenait constante après quelques jours de séchage. La teneur en eau (TE) est exprimée en pourcentage de la masse sèche:

$$
T E=\frac{(M F-M S)}{M S} \times 100
$$

\section{Microscopie électronique à balayage}

Seules les aiguilles de l'année 1987 ont été observées en microscopie électronique. Les ai- 
guilles sont prélevées comme indiqué plus haut. Après séchage à l'étuve à $60^{\circ} \mathrm{C}$ pendant une semaine, de préférence à une déshydratation (Sauter et Voss, 1986), elles sont stockées au sec jusqu'au moment de leur préparation. Elles sont alors disposées sur un portè-échantillon en aluminium où elles sont fixées à l'aide d'une colle conductrice au carbone (LEIT-C de Balzers). Les échantillons sont ensuite recouverts d'une fine couche de carbone (environ $10 \mathrm{~nm}$ ) au moyen d'un évaporateur CED 020 Balzers de manière à assurer leur conductivité électrique. Les observations ont été conduites sur une centaine d'aiguilles, des classes de dépérissement 1 et 3 , avec des grandissements voisins de 600 et avec une tension d'accélération égale à $25 \mathrm{kV}$ à l'aide d'un microscope à balayage Stereoscan 90B (Cambridge Instruments).

Pour décrire quantitativement les modifications de l'état de surface des aiguilles au niveau des cires, nous avons utilisé les critères définis par Sauter et Voss (1986) :

- état I, pas de dégradation ou d'agrégation des cires épistomatiques ou épicuticulaires;

- état II, début d'agrégation des cires épistomatiques (sur moins de 1/3 de la surface des sto- mates) et début de dégradation et d'agglomération des cires épicuticulaires (disparition des structures cireuses inférieure à $30 \%$ );

- état III, agrégation de moyenne à forte des cires épistomatiques sur $1 / 3$ a $8 / 10$ de la surface des stomates, dégradation et disparition de 30 à $80 \%$ des cires épicuticulaires;

- état IV, agrégation très importante des cires épistomatiques (formation d'une couche continue de cire sur presque $9 / 10$ de la surface des stomates), disparition presque totale des cires épicuticulaires.

\section{RÉSULTATS}

\section{Contenu hydrique des aiguilles}

Dans la figure 1 sont consignées, pour chacune des stations et pour l'ensemble de l'échantillon, les variations de la teneur moyenne en eau des aiguilles des 4 générations en fonction de l'état de défoliation

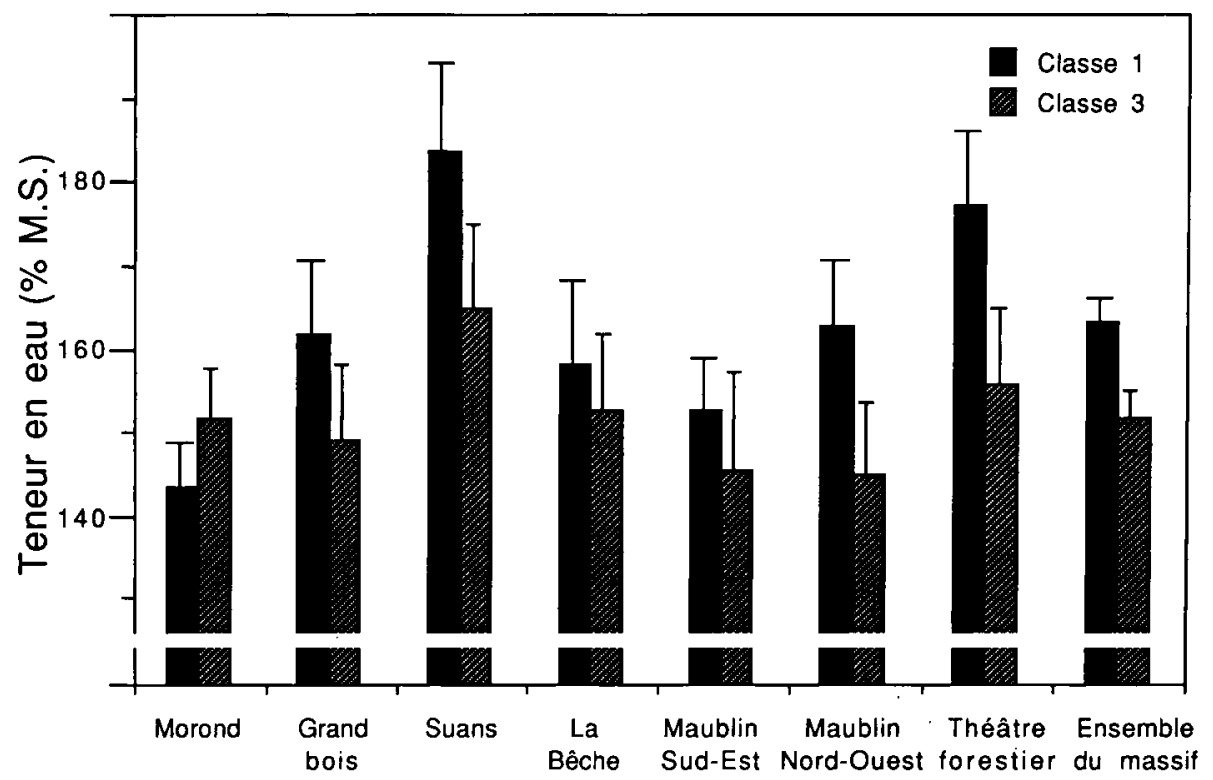

Fig 1. Variations de la teneur moyenne en eau des aiguilles de l'ensemble des générations dans les différentes stations en fonction de l'état de défoliation des arbres. L'intervalle de confiance est fourni pour $P=0,05$. 
des arbres. Quelle que soit la classe de dépérissement considérée, la teneur moyenne en eau est liée à l'origine géographique des aiguilles. II existe par exemple des différences statistiquement significatives entre les valeurs les plus élevées enregistrées pour les arbres sains dans les stations de Suans $(183,5 \pm 11,1 \%$ $M S)$ et de Théâtre forestier $(177,3 \pm 9,6 \%$ MS) et les valeurs les plus faibles présentées à Morond $(143,3 \pm 6,1 \% M S)$ et à Maublin Sud-Est $(152,6 \pm 7,2 \% M S)$.

En règle générale, la teneur moyenne en eau des aiguilles est fonction de l'état de défoliation : à l'exception des valeurs enregistrées pour la station de Morond, les teneurs moyennes en eau des aiguilles des arbres de classe 3 sont inférieures à celles des aiguilles des arbres de classe 1. Ces différences sont statistiquement significatives dans 3 des stations étudiées (Suans, Maublin Nord-Ouest et Théâtre forestier). A l'échelle de l'ensemble de l'échantillon étudié, la teneur moyenne en eau des aiguilles des arbres de classe 1 $(163,3 \pm 3,6 \%$ MS) est aussi statistiquement supérieure à celle des arbres de classe $3(151,6 \pm 3,4 \% M S)$. Ceci est également vrai quelle que soit la génération d'aiguilles considérée : à âge égal, les teneurs moyennes en eau des aiguilles des arbres présentant une défoliation importante sont toujours statistiquement inférieures à celles des arbres non défoliés (fig 2). II est important de remarquer que les aiguilles des arbres dépérissants montrent, à un âge donné, des teneurs moyennes en eau normalement présentées par les aiguilles des arbres sains lorsqu'elles sont plus âgées. Si l'on considère par exemple les aiguilles de l'année 1986 des arbres de classe 3 , on constate que leur contenu hydrique, qui est de l'ordre de $150 \%$ MS, n'est pas significativement différent de celui des aiguilles mises en place un an plus tôt c'est-à-dire en 1985 par les arbres de classe 1.

\section{Observation des surfaces foliaires en microscopie électronique à balayage}

La figure 3 fournit des exemples caractéristiques d'images des surfaces foliaires telles qu'elles ont été obtenues au cours d'une centaine d'observations. Les aiguilles des arbres de classe 1 (fig 3a) présentent la structure classique (état l) : la surface foliaire est hérissée de cires épicuticulaires en forme d'aiguilles. Au niveau du stomate et à sa périphérie, les cires stomatiques sont abondantes et nettement individualisées.

Chez les arbres de classe 3 (fig $3 b$ ), ce feutrage de cires stomatiques a pratiquement disparu et seul un agglomérat de cires mal différenciées est visible. Les

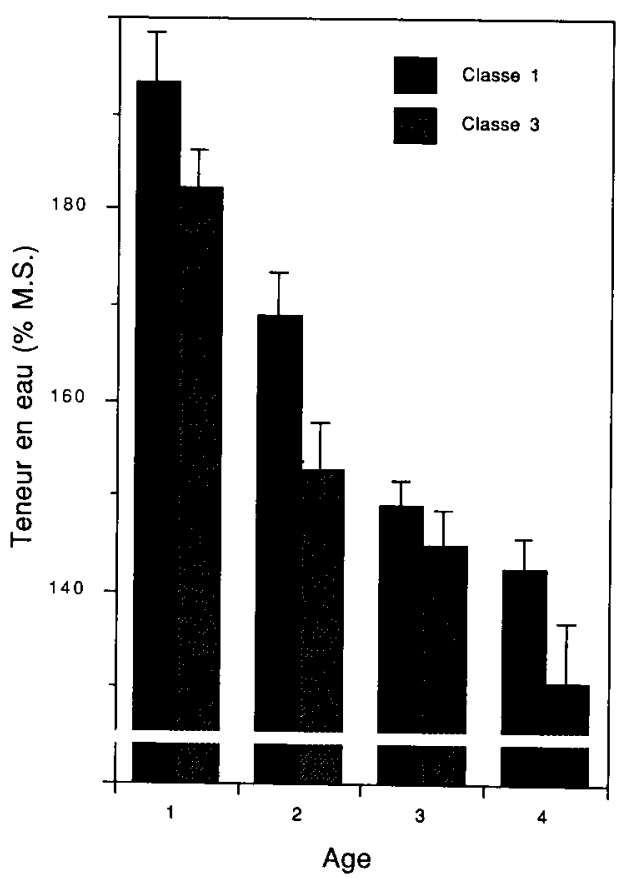

Fig 2. Variations de la teneur moyenne en eau des aiguilles en fonction de leur âge et en fonction de l'état de défoliation des arbres de l'ensemble des stations. L'intervalle de confiance est donné pour $P=0,05$. 

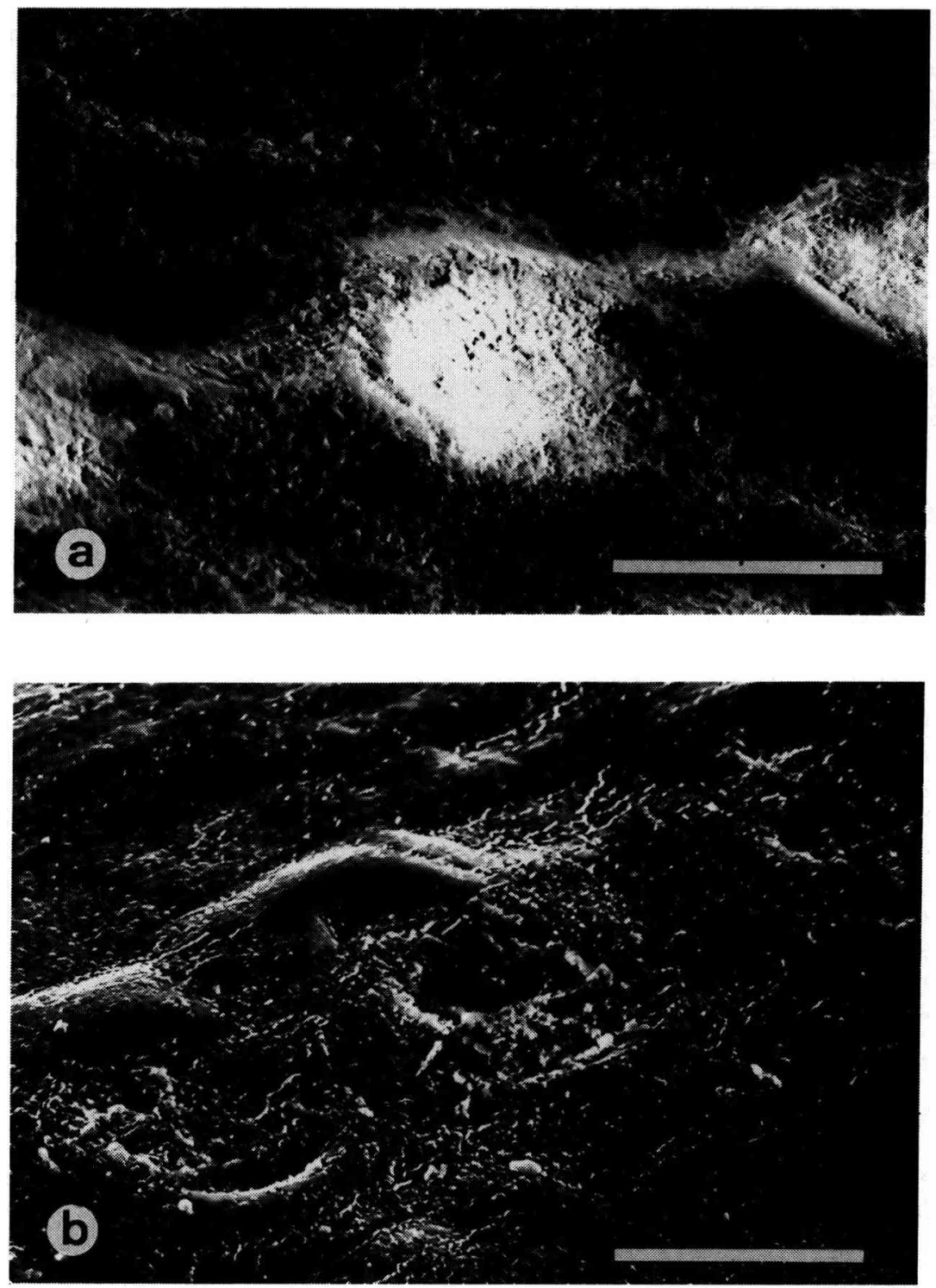

Flg 3. Surfaces foliaires observées en microscopie électronique à balayage. Les barres horizontales représentent $50 \mu \mathrm{m} ; a$, aiguilles de l'année d'arbres de classe 1; b, aiguilles de l'année d'arbres de classe 3. 
cires épicuticulaires sont pratiquement absentes et la surface cuticulaire ne présente plus que quelques structures mal individualisées. Ce niveau d'altération des cires à la surface des aiguilles des épicéas dépérissants correspond à l'état III tel qu'il a été défini à partir des critères de Sauter et Voss (1986). Dans le cadre de cette expérience, ces symptômes ont été retrouvés sur plus de $80 \%$ des échantillons observés : quelle que soit l'origine géographique des aiguilles, il existe toujours une relation nette entre l'état de défoliation des arbres et l'aspect des surfaces foliaires.

\section{DISCUSSION}

Quelle que soit la classe de dépérissement considérée, les teneurs moyennes en eau des aiguilles des épicéas du Jura présentent d'importantes variations en fonction de l'origine géographique des arbres (fig 1). Ces variations de teneur moyenne en eau enregistrées entre arbres de différentes stations montrent que ce paramètre dépend de facteurs stationnels dont on ne peut encore préciser la nature. Bien que la teneur moyenne en eau des aiguilles des arbres de classe 3 soit inférieure à celle des arbres de classe 1 , ce paramètre ne peut donc être utilisé comme un indicateur de l'état de dépérissement d'un arbre ou d'un petit nombre d'arbres. Néanmoins, à l'échelle de l'ensemble de l'échantillon étudié, la teneur moyenne en eau des aiguilles est étroitement liée à l'état de défoliation des arbres et elle pourrait de ce fait constituer un moyen fiable pour caractériser la vitalité d'un peuplement. Ces résultats sont en accord avec ceux de Mehlhorn et al (1988), Badot et al (1988), Rosenkranz et al (1989).

Quel que soit l'état de défoliation des arbres, le contenu hydrique des aiguilles diminue avec l'âge de celles-ci. Ceci est sans doute lié aux rôles spécifiques joués par les différentes générations d'aiguilles : l'essentiel de l'activité photosynthétique est assurée par les aiguilles de l'année, alors que les aiguilles plus âgées auraient un rôle de réserve (Prinz et Krause, 1987). Les aiguilles des arbres dépérissants montrent à un âge donné des teneurs moyennes en eau égales à celles normalement enregistrées environ un an plus tard dans les aiguilles des arbres sains. Un résultat similaire a été obtenu précédemment concernant l'évolution du $\mathrm{pH}$ vacuolaire au cours de vieillissement des aiguilles (Badot et Badot, 1989). Chez les arbres dépérissants, les aiguilles atteindraient plus rapidement un état physiologique caractérisant les aiguilles plus âgées. Ainsi, la défoliation pourrait être liée à un vieillissement prématuré des aiguilles. Une telle sénescence précoce a déjà été évoquée dans des travaux ayant trait à l'étude du dépérissement (Garrec et al, 1989). Les informations recueillies ici ne permettent toutefois pas de déterminer si cette baisse précoce de la teneur moyenne en eau correspond à une diminution de la masse d'eau ou à une augmentation de la biomasse sèche. Une moindre croissance peut en effet se traduire par des cellules plus petites à vacuole réduite et par un rapport masse d'eau/MS plus faible sans qu'aucune contrainte hydrique n'ait été exercée. Même si les résultats de l'étude dendrochronologique (Badot et al, 1990) montre une corrélation entre l'actuel épisode de dépérissement et les sécheresses qui ont affecté l'Europe au cours des dernières années, d'autres investigations, par exemple des mesures de teneur en eau relative et de potentiel hydrique réalisées à différents moments de l'année, devront être effectuées afin de déterminer si le fonctionnement hydrique des épicéas dépérissants du Jura est perturbé ou non. 
Les observations en microscopie électronique à balayage des surfaces foliaires révèlent l'existence chez les arbres dépérissants de modifications de l'abondance, de l'aspect et de la distribution des cires épicuticulaires. De telles altérations ont déjà été signalées dans la littérature (Tuomisto, 1988; Gunthardt-Goerg et Keller, 1987; Barnes et al, 1988). Les perturbations métaboliques induites par divers agents polluants (Percy et Baker, 1987) se répercuteraient sur la formation de la cuticule dans les jeunes aiguilles (Garrec et Kerfourn, 1989). Chez des arbres soumis à ces agents, la mise en place des cires cuticulaires et stomatiques serait affectée, ce qui provoquerait une augmentation de la perméabilité des cuticules vis-à-vis de l'eau. Un argument supplémentaire à l'appui de cette conception est constitué par les résultats récents obtenus lorsque des aiguilles sont soumises à des brouillards acides (Mengel et al, 1989) ou à un mélange de brouillard acide et d'ozone (Barnes et al, 1990), la transpiration cuticulaire est alors toujours plus élevée dans les aiguilles traitées. De tels processus pourraient conduire à une diminution de la quantité d'eau présente dans les aiguilles et pourraient ainsi rendre compte de la baisse de teneur moyenne en eau des arbres dépérissants. Des cinétiques de déshydratation d'aiguilles excisées, qui donnent de bonnes indications sur la transpiration cuticulaire (Mengel et al, 1989), ou des mesures de conductance foliaire à l'aide d'un poromètre devront être réalisées sur notre matériel pour confirmer la validité de cette hypothèse.

A ce stade de notre travail, il importe de poursuivre nos investigations afin de déterminer quelle est l'origine des symptômes réduction des teneurs en eau et altération des cuticules - identifiés chez les épicéas dépérissants du Jura. II convient en particulier de préciser la nature du phénomène de sénescence précoce des aiguilles et de rechercher si les relations hydriques sont perturbées chez les épicéas dépérissants et quelles peuvent alors être les conséquences sur la croissance et le développement de la plante (Schulze, 1986; Mac Intyre, 1987).

\section{REMERCIEMENTS}

Les auteurs adressent leurs plus vifs remerciements a MJ Badot pour son efficace collaboration, à P Lacroix directeur du Service Graines et Plants de l'Office National des Forêts, ainsi qu'aux équipes de grimpeurs de cet organisme, pour leur disponibilité et leur compétence lors de la collecte des échantillons.

Ce travail a bénéficié de soutiens financiers du Conseil Régional de Franche-Comté et du programme DEFORPA.

\section{RÉFÉRENCES}

Badot PM, Badot MJ (1989) Contribution à l'analyse des caractères physiologiques des épicéas dépérissants (Picea abies $L$ ) du Jura. Ann Sci Univ Besançon Biol Ecol 5, 39-44

Badot PM, Garrec JP, Millet B, Badot MJ, Mercier J (1988) Dépérissement et état hydrique des aiguilles chez le Picea abies. Can J Bot 66, 1693-1701

Badot PM, Perrier P, Badot MJ (1990) Dépérissement, déficit hydrique et variations de croissance radiale chez le Picea abies. Can J Bot 68, 1536-1541

Barnes JD, Davison AW, Booth TA (1988) Ozone accelerates structural degradation of epicuticular wax on Norway spruce needles. New Phytol 110, 309-318

Barnes JD, Eamus D, Brown A (1990) The influence of ozone, acid mist and soil nutrient status on Norway spruce (Picea abies L Karst). I. Plant water relations. New Phytol $114,713-720$

Becker M (1989) The role of climate on present and past vitality of silver fir forests in the Vosges mountains of northeastern France. Can J For Res 19, 1110-1117 
Bruckert S, Tan BS (1986) Etude du dépérissement des forêts de l'étage montagnard du Jura en relation avec les sols calciques ou acides du karst, l'importance de leurs éléments nutritifs et l'état nutritionnel des peuplements. Ann Sci Univ Besançon Biol Vég 6. 37-44

Buffet M (1987) Le réseau de surveillance des forêts. Description. Problématique. In: Les recherches en France sur le dépérissement des forêts. Programme DEFORPA, $1^{\text {er }}$ Rapport. ENGREF, 11-16

Garrec JP, Kerfourn C (1989) Effets des pluies acides et de l'ozone sur la perméabilité à l'eau et aux ions de cuticules isolées. Implication dans le phénomène de dépérissement des forêts. Environ Exp Bot 29, 215-228

Garrec JP, Richardin I, Le Maout L, Rose C, Gérard B, Mathieu Y (1989) Etude du pH et du pouvoir tampon des aiguilles de sapins (Abies alba $L$ ) et d'épicéas (Picea abies $L$ ) dépérissants. Application en symptomatologie? Ann Sci For 46, 55-69

Guehl JM (1985) Etude comparée des potentialités hivernales d'assimilation carbonée de trois conifères de la zone tempérée (Pseudotsuga menziesii Mirb, Abies alba Mill, et Picea excelsa Link). Ann Sci For 42, 23-38

Gunthardt-Goerg MS, Keller T (1987) Some effects of long-term ozone fumigation on Norway spruce. II. Epicuticular wax and stomata. Trees 1, 145-150

Landmann G, Bonneau M, Adrian M (1987) Le dépérissement du sapin pectiné et de l'épicéa commun dans le massif vosgien est-il en relation avec l'état nutritionnel des peuplements? Rev For Fr (Nancy) 39, 5-11

Lévy $G$, Becker $M$ (1987) Le dépérissement du sapin dans les Vosges : rôle primordial de déficits d'alimentation en eau. Ann Sci For 44, 403-416
McIntyre GI (1987) The role of water in the regulation of plant development. Can J Bot 65, 1287-1298

Mehlhorn H, Francis BJ, Wellburn AR (1988) Prediction of the probability of forest decline damage to Norway spruce using three simple site-independent diagnostic parameters. New Phytol 110, 525-534

Mengel K, Hogrebe AMR, Esch A (1989) Effect of acidic fog on needle surface and water relations of Picea abies. Physiol Plant 75, 201207

Ministère de l'Agriculture (1988) Etat sanitaire des arbres forestiers. Observations 1987, 1-6

Percy KE, Baker EA (1987) Effects of simulated acid rain on production, morphology and composition of epicuticular wax and on cuticular membrane development. New Phytol 107, 577-589

Prinz B, Krause GHM (1987) Le dépérissement des forêts en République Fédérale d'Allemagne. Pollut Atmos 113, 45-53

Rehfuess KE (1987) Perceptions of forest diseases in Central Europe. Forestry (Oxf) 60, $1-11$

Rosenkranz T, Bareis C, Braun J, Wild A (1989) Investigation on the water status of spruce trees in relation to the occurrence of novel forest decline. Plant Physiol Biochem 27, 333340

Sauter JJ, Voss JU (1986) SEM - Observations on the structural degradation of epistomatal waxes in Picea abies (L) Karst - and its possible role in the "Fichtensterben". Eur J For Pathol 16, 408-423

Schulze ED (1986) Whole-plant responses to drought. Aust J Plant Physiol 13, 127-141

Tuomisto H (1988) Use of Picea abies needles as indicators of air pollution: epicuticular wax morphology. Ann Bot Fenn 25, 351-364 